\title{
Improving GFDM Symbol Error Rate Performance using "Better than Nyquist" Pulse Shaping Filters
}

\author{
Atul Kumar, Maurizio Magarini, Member, IEEE, Stefano Bregni, Senior Member, IEEE
}

Politecnico di Milano, Dept. of Electronics, Information and Bioengineering, P.za Leonardo Da Vinci 32, 20133 Milano, ITALY

\begin{abstract}
Fourth generation (4G) cellular systems have been optimized to provide high data rates and reliable coverage to mobile users. New waveforms at the physical layer are needed. Generalized frequency division multiplexing (GFDM) is a candidate modulation for the fifth generation (5G) standard based on multi-branch multicarrier filter bank approach. A main characteristic of GFDM is its low out of band emission, achieved by means of a flexible time-domain pulse shaping of individual subcarriers. In this paper, the influence of the improved "Better than Nyquist" pulse shaping filters on symbol error rate (SER) performance of the GFDM system in the case of zero forcing $(\mathrm{ZF})$ receiver is investigated. We considered their use in GFDM to evaluate the impact on SER performance in case of 16-QAM transmission over an additive white Gaussian noise channel. Moreover, we also considered the concept of the wavelet for better time-frequency localization of the pulse shaping filters by using the Meyer auxiliary function. Numerical results are reported to demonstrate the superior SER performance achieved.

Keywords - 4G cellular systems; FDM.
\end{abstract}

\section{INTRODUCCIÓN}

Hoy en día, hay un crecimiento de la demanda de dispositivos inteligentes con: capacidades superiores al ritmo de transmisión de la 4ta Generación (4G), ultra-bajo consumo eléctrico de las baterías de los sensores de comunicación, tiempo muy corto de la respuesta y una ultra-alta fiabilidad de las aplicaciones de control. Estos aparecen como factores decisivos para la implementación de los futuros sistemas de comunicación [1]. Por estas razones la próxima red celular de 5 ta generación $(5 \mathrm{G})$ tiene que ser capaz de hacer frente al alto número de dispositivos conectados, así como también, a un crecimiento significativo del volumen de datos [2].

Los principales escenarios para las redes celulares $5 \mathrm{G}$ son: El Internet de las Cosas (internet of things, IoT), comunicación de tipo maquina (machine type communication, MTC) [3], Internet Táctil [4], Comunicaciones Bitpipe [2] y la red de área regional inalámbrica (wireless regional area network, WRAN) [5]. Los requerimientos de estas aplicaciones necesitan de nuevas formas de onda, cuyas señales están más localizadas en el tiempo y la frecuencia y además implican más requerimientos sincronizados, los cuales a su vez reducen los gastos generales de la red y, por lo tanto, también la del retardo comparado con el 4G. El uso de nuevas formas de onda deben ser una agregación de portadores más factibles que con la OFDM. Además la MTC se caracteriza por dispositivos con accesos aleatorios esporádicos que pueden ser alcanzados mediante técnicas de radio cognitiva (cognitive radio, CR) [6], donde la flexibilidad en la conformación y la estructura de la señal transmitida es un aspecto fundamental. En el caso del Internet Táctil, un ultrabajo retardo y un corto periodo de fiabilidad de la trama son requisitos esenciales. En el caso de una red WRAN, una larga área de cobertura y un gran retardo de propagación son los mayores desafíos. Finalmente, en el caso de comunicaciones Bitpipe los mayores requisitos son redes densas con menor interferencia.

Un sistema CR debe ser capaz de agregar espectro en espacios vacios, donde al mismo tiempo, la fuga del, fuera de banda (out-of-band, OOB) se minimiza con el objetivo de no afectar a los sistemas cercanos. Como se sabe, los sistemas de transmisión celular actuales como el de la evolución a largo plazo (long-term evolution, LTE) y LTE-avanzado (long-term evolution advanced, LTE-A) emplean la OFDM en el nivel físico [7]. Aunque la OFDM es robusta contra el canal de desvanecimiento a frecuencia selectiva y ofrece una eficiente implementación usando la Transformada Rápida de Fourier, no es adecuada para los futuros requisitos [8]. Las principales debilidades de la OFDM son su sensibilidad hacia el desplazamiento de la frecuencia portadora y el ruido de fase, y a la fuga de su larga OOB, la cual la hace poco atractiva para las aplicaciones CR. Mientras los efectos del desplazamiento de la frecuencia portadora y del ruido de fase, pueden ser reducidos a través de una sincronización fina y de unas funciones de seguimiento de la fase, se puede obtener una reducción de la emisión de la OOB a través de una conformación de pulso de las sub-portadoras individuales en el dominio del tiempo. En efecto, una alta radiación de OOB de la OFDM de debe al filtro de conformación de pulso rectangular, que se aplica a cada sub-portadora en el domino del tiempo, la cual implica grandes colas en el dominio de la frecuencia. Otro problema de la OFDM es el requisito de un prefijo cíclico (cyclic prefix, CP) en cada símbolo, el cual reduce la eficiencia del espectro.

Un estudio de literatura revela que la formas de onda propuestas para el nivel físico del $5 \mathrm{G}$ puede ser categorizado en dos grupos, algunos con conformación de pulso lineal, [9], [10], y otros con conformación de pulso circular, [11], [12]. Entre estas formas de onda, hay las de tipo, Multiportador de Banco de Filtros (filter bank multicarrier, FBMC) [9], las cuales se basan en la conformación de pulso lineal, y las de tipo Multiplexación por División de Frecuencia Generalizada (generalized frequency división multiplexing, GFDM) [11], la cual se basa en la conformación de pulso circular. Estas son las candidatas más prometentes para las aplicaciones del emergente 5G. En la modulación FBMC el uso de un prototipo de filtro de conformación de pulso sobre cada sub-portadora se propone para minimizar la radiación OBB. Varios trabajos se centran en el diseño de los filtros de conformación de pulso para los FBMC con el objetivo de minimizar la radiación OOB [13] [14]. 
La principal propiedad del FBMC es la de mantener la ortogonalidad entre las sub-portadoras y la de permitir la reducción de las emisiones del OOB a través de elección adecuada de los filtros de conformación de pulso. Sin embargo, no tiene otras propiedades que son necesarias para el $5 \mathrm{G}$ como la robustez contra el canal de desvanecimiento a frecuencia selectiva. En cambio, la GFDM que deriva de un planteamiento de banco de filtros, se basa en un esquema de Modulación Multiportadora (MCM) de bloques filtrados y permite alcanzar una alta robustez contra el canal de desvanecimiento a frecuencia selectiva. La transmisión de datos en cada bloque está distribuida en el tiempo y la frecuencia, y cada sub-portadora está formada por impulsos. La reducción de la emisión de la OOB se obtiene en la GFDM usando diferentes filtros de conformación de pulso en el dominio del tiempo aparte del convencional filtro rectangular usado en la CP-OFDM [15]. Además, la técnica, tail biting, usada en la GFDM hace que la longitud del CP sea independiente respecto a la longitud de los filtros de transmisión y recepción [13]. Esto permite reducir la sobrecarga causada por el CP y por lo tanto aumentar la eficiencia del espectro. Otras ventajas de la GFDM están relacionadas con su flexibilidad a través del cambio de número de segmentos temporales o de sub-portadoras en una trama del GFDM, eso puede cubrir las convencionales CP-OFDM y la del acceso múltiplo por dominio de frecuencia de tipo monoportadora [1]. Además, la GFDM permite alcanzar una frecuencia adicional, al precio de una autointerferencia inherente [16], que se origina desde la no ortogonalidad, y por lo tanto prohíbe el desacoplamiento de las sub-portadoras individuales. Estas características hacen de la GFDM la candidata más fuerte con un esquema MCM para el 5G [17].

La principal contribución de este artículo, consiste en la evaluación del rendimiento del SER del GFDM usando diferentes transmisiones de filtros de conformación de pulso de Nyquist, sobre un canal de ruido blanco gaussiano aditivo (additive white Gaussian noise, AWGN). Aquí, consideramos impulsos de tipo Xia, familia propuesta en la [18], y extensiones de esta familia de impulsos, propuesta en la [19] con el nombre "Better than Nyquist", donde presentamos el objetivo de alcanzar una alta tolerancia, contra el error temporal del símbolo, en un esquema de modulación monoportadora. Como sugerido en la [20], cuando los filtros de conformación de pulsos de Nyquist se consideran en la GFDM, se puede alcanzar una mejora en el rendimiento, a través de un intercambio de rol, de la variable independiente de la frecuencia con la variable independiente del tiempo, en la expresión formal, definiendo el pulso en coseno alzado. La principal ventaja del intercambio de variable tiempofrecuencia es que no hay superposición en el dominio de la frecuencia y por lo tanto se observa menos interferencia.

El artículo está organizado en el modo siguiente. La Sección II ofrece una breve descripción de los modelos del sistema GFDM junto con las notaciones que usaremos en este artículo. Los diferentes tipos de filtros de conformación de pulso de Nyquist mejorado se presentan en la Sec. III, mientras el análisis del SER del GFDM se verá en la Sec. IV. Los resultados de la simulación por diferentes filtros de conformación de pulsos se presentan en la Sec. V y, finalmente, las imágenes se muestran en las Sec. VI.

\section{MODELO DEL SISTEMA}

El diagrama de bloques del esquema de transmisión GFDM considerado, Se da en la [17]. En este esquema la parte transmisora incluye varios bloques. Usando una notación matricial, el vector de bits de entrada $\mathbf{b}$ se aplica en la entrada del mapeador que produce un vector de datos d cuyas $N$ entradas toman valores de constelaciones complejas, donde consideramos la QAM. El diagrama de bloques de la matriz del modulador del GFDM Se da en la [17]. El alto flujo de la tasa de error se aplica en la entrada del modulador GFDM que divide la secuencia de entrada en $\mathrm{K}$ grupos de $\mathrm{M}$ símbolos con una baja tasa de error como

$$
\mathbf{d}=\left[\left(\boldsymbol{d}_{0}\right)^{T},\left(\mathbf{d}_{1}\right)^{T}, \ldots \ldots,\left(\mathbf{d}_{K-1}\right)^{T}\right]^{T},
$$

donde $\mathbf{d}_{k}=\left[d_{k, 0}, d_{k, 1}, \ldots ., d_{k, M-1}\right]^{T}$,

siendo $d_{k, m}$ el $m$-ésimo sub-símbolo transmitido del QAM en la $k$-ésima sub-portadora y $(.)^{\mathrm{T}}$ es un operador. La duración de cada bloque de datos $\mathbf{d}_{k}$ es $M T_{\mathrm{s}}$ y el intervalo de la subportadora es igual a $1 / M T_{\mathrm{s}}$. Cada símbolo complejo $d_{k, m}$ modula en amplitud la respuesta impulsiva

$$
g_{k, m}[n]=g[(n-m K) \bmod N] e^{\frac{-j 2 \pi k n}{K}},
$$

donde $g[n]$ es un prototipo de respuesta impulsiva discreta, mod denota la operación modulo $\mathrm{N}$ y $\mathrm{n}$ es el índice del muestreo. Cabe observar que, cada $g_{k, m}[n]$ corresponde a una versión traslada del tiempo y de la frecuencia, donde la exponencial compleja, ejecuta la operación de traslado en el dominio de la frecuencia. La $n$-ésima entrada del $M K \times 1$ del vector transmitido $\mathbf{x}$, se da por

$$
x[n]=\sum_{k=0}^{K-1 M-1} \sum_{m=0} d_{k, m} g_{k, m}[n], \quad n=0,1, \ldots \ldots . . . K M-1 .
$$

El vector $\boldsymbol{g}_{k, m}=\left[g_{k, m}[0], g_{k, m}[1], \ldots, g_{k, m}[M K-1]\right]^{T}$ colecciona las muestras de $g_{k, m}[n]$. Aquí, representamos la ecuación (2) en su forma matricial como

$$
\mathbf{x}=\mathbf{A d},
$$

donde $\mathbf{x}=[x[0], x[1], \ldots, x[M K-1]]^{T} \mathrm{y}$

$$
\mathbf{A}=\left[\begin{array}{llll}
\boldsymbol{g}_{0,0} \ldots \boldsymbol{g}_{K-1,0} & \boldsymbol{g}_{0,1} \ldots . . \boldsymbol{g}_{K-1,1} & \boldsymbol{g}_{0, M-1} \ldots . \boldsymbol{g}_{K-1, M-1}
\end{array}\right] .
$$

La matriz $M K \times M K$. Antes de la transmission una CP de longitud $N_{c p}$ se agrega a la forma del vector

$$
\hat{\boldsymbol{x}}=\left[\mathbf{x}\left(N-N_{C P}: N-1\right)^{T}, \mathbf{x}^{T}\right]^{T} .
$$

Considerando la transmission sobre un canal AWGN, la señal recibida se modela como

$$
\hat{\boldsymbol{y}}=\hat{\boldsymbol{x}}+\mathbf{w},
$$

donde cada entrada de w es una variable aleatoria Gaussiana compleja independiente e idénticamente distribuidas con media cero y con varianza $\sigma_{\mathrm{w}}{ }^{2}$ Donde, el vector recibido después de la eliminación de la $\mathrm{CP}$ se define como

$$
\mathbf{y}=\hat{\boldsymbol{y}}\left(N_{C P}: N_{C P}+N-1\right) .
$$

Algunas opciones del receptor estándar se pueden usar para la demodulación de la señal GFDM:

- Filtro adaptado (matched filter, MF): El receptor MF maximiza la relación señal/ruido por cada sub-portadora, pero con el efecto de introducir autointerferencia cuando se 
aplica un pulso transmitido no ortogonal. Los métodos para la eliminación de la autointerferencia, a través de un receptor $\mathrm{MF}$, se proponen en la [16], donde se muestra que, el rendimiento casi igual al MCM ortogonal, puede ser alcanzado. El mismo receptor MF se considera en la [15] para estudiar el impacto de los diferentes filtros de conformación de pulso sobre la OOB y el rendimiento de la tasa de error.

- Error cuadrático medio mínimo lineal (minimum meansquared error, MMSE): El receptor MMSE produce una mejora de la compensación entre la autointerferencia y la intensificación del ruido.

- Zero forcing (ZF): El receptor ZF remueve completamente la autointerferencia en el lado receptor, a costa de introducir una posible intensificación del ruido, la cual depende de la respuesta impulsiva del filtro de conformación de pulso [17]. La intensificación del ruido tiene un impacto en el rendimiento de la tasa de error (SER) del símbolo. Es entonces que a través de un diseño apropiado de la conformación de pulsos, es posible superar el efecto de la ampliación del ruido y mejorar el rendimiento del SER.

Aquí, consideramos el receptor $Z F$, la matriz $\mathbf{B}_{Z F}=\mathbf{A}^{-1}$, el vector de la señal recibida, dados como

$$
\mathbf{z}=\mathbf{B}_{Z F} \mathbf{A d}+\mathbf{B}_{Z F} \mathbf{w}=\mathbf{d}+\tilde{\mathbf{w}} .
$$

De la (6) observamos que el receptor $\mathrm{ZF}$ elimina completamente la autointerferencia al precio de aumentar la intensificación del ruido $[2,17]$. El factor de intensificación del ruido (noise enhancement factor, NEF) $\xi$ determina la reducción del SNR cuando se usa el receptor ZF. Esto se define como

$$
\xi_{k}=\sum_{n=1}^{M K}\left|\mathbf{B}_{Z F}(k, n)\right|^{2}=\xi,
$$

el cuál es el mismo por cada $k$ [2].

\section{FILTRO DE CONFORMACIÓN DE PULSO}

La elección de la respuesta impulsiva de la conformación de pulso discreta $g[n]$ influencia fuertemente las propiedades del espectro de la señal GFDM. Tal respuesta impulsiva puede ser obtenida tomando muestras de una respuesta impulsiva de tiempo continuo $g(t)$ mostrada como

$$
g_{w}(t)=\left\{\begin{array}{lr}
g_{\text {wdown }}(t) & 1 \leq t \leq K T s \\
g_{\text {wup }}(t) & M \times K-K \leq t \leq M \times K \\
1 & \text { otherwise }
\end{array}\right.
$$

donde $g_{\text {wup }}(t)=g_{\text {pulse }}(t) \quad$ y $g_{\text {wdown }}(t)=1-g_{\text {pulse }}(t)$ donde $g_{\text {pulse }}(t)$ representa el diferente tipo de filtro de conformación de pulso como presentado debajo. Como es sabido, la respuesta impulsiva de Nyquist tiene valores nulos periódicos en el dominio del tiempo, en los múltiplos enteros del periodo del símbolo $T_{s}$. Cuando nos movemos en el dominio de la frecuencia esto se convierte en una simetría vestigial [20]. Esto significa que su espectro exhibe simetrías impares alrededor de la frecuencia de corte, la cual es la mitad de la tasa del símbolo. Por lo tanto, los filtros de Nyquist, se pueden definir, imponiendo una simetría impar alrededor de la frecuencia de corte. En los sistemas de comunicación, el filtro de Nyquist global, esta generalmente dividida en dos partes, en un filtro de
Nyquist de tipo raíz cuadrada en el transmisor y en un filtro de Nyquist de tipo raíz cuadrada en el receptor. Luego, la condición de simetría se satisface por los cuadrados de los coeficientes frecuenciales. Como propuesto en la [20], con el objetivo de dibujar un apropiado filtro de Nyquist para ser usado en la GFDM, las principales simetrías impares necesitan ser aplicadas desde una perspectiva del dominio del tiempo, intercambiando el rol de la variable independiente de la frecuencia con aquella independiente del tiempo en la función analítica, definiendo el pulso de Nyquist. La ventaja que deriva desde este intercambio formal entre las dos variables independientes es la eliminación de la superposición en el dominio del tiempo con la consecuente reducción de las interferencias. Esta operación será hecha para todos los filtros de conformación de pulso considerados en esta Sección.

El concepto de ondícula está incluida aquí también, para alcanzar una mejor localización tiemporal-frecuencial y también para aumentar la flexibilidad en el diseño del filtro de conformación de pulso. Como es sabido, el pulso de Nyquist de tipo raíz cuadrada tiene un mayor impacto en el diseño del sistema y por lo tanto, una solución alternativa es definirlo en el dominio del tiempo. Esta solución alternativa debe asegurar que la derivada del pulso diseñado sea suave, para limitar la gama de la frecuencia como sea posible. Muchos autores han investigado soluciones basadas en el concepto de ondícula [22], [23]. Una ondícula es una fluctuación ondulatoria cuya amplitud inicia desde cero, crece y luego decrece hasta llegar a cero. Para asegurar esta suave transición, Meyer ha introducido una función auxiliar en la [23] y, como propuesto en la [20], puede ser usada como un argumento interno de diferentes filtros de conformación de pulso. La función auxiliar de Meyer se define como

$$
v(x)=\left\{\begin{array}{cc}
0, & x \leq 0 \\
x^{4}\left(35-84 x+70 x^{2}-20 x^{3}\right) & 0<x<1 \\
1, & x \geq 1
\end{array}\right.
$$

Por eso, en nuestros resultados, consideraremos el impacto en el rendimiento del SER de los filtros de conformación de pulsos de Nyquist mejorados, con y sin la función auxiliar de Meyer en el caso de la transmisión en un canal AWGN.

\section{A. Root ramp}

Esta es una categoría base de filtros de conformación de pulsos definida por funciones de tipo root ramp en el dominio del tiempo, determinado por un especifico factor de roll-off $\alpha$ [2]. La expresión en el dominio del tiempo, que resulta del intercambio de la variable frecuencial independiente con la del tiempo, es

$$
g_{\text {Ramp }}(t)=\left\{\begin{array}{cc}
1, & |t| \leq \frac{(1-\alpha) T}{2} \\
\frac{1}{2}\left[1+P_{\text {Ramp }}(t)\right], \frac{(1-\alpha) T}{2} & <|t| \leq \frac{(1+\alpha) T}{2} \\
0, & \text { otherwise }
\end{array}\right.
$$

donde $T=K T_{s}$ y $P_{\text {Ramp }}(\mathrm{t})$ es el argumento interno, escrito como

$$
P_{\text {Ramp }}(t)=\frac{|t|-\frac{(1-\alpha) T}{2}}{\alpha T}
$$

Introduciendo la función auxiliar de Meyer $v(x)$ el argumento 
interno tiene que ser modificado como

$$
P_{\text {Ramp }}(t)=v \cdot \frac{|t|-\frac{(1-\alpha) T}{2}}{\alpha T}
$$

El filtro de conformación de pulso de tipo root ramp se define como

$$
g_{\text {Root Ramp }}(t)=\sqrt{g_{\text {Ramp }}(t)}
$$

\section{B. Root raised cosine ( $R R C)$}

Esta categoría de filtros de conformación de pulsos, se define por las funciones bien conocidas, coseno alzado (raised cosine, RC) y el root raised cosine (RRC), en el dominio del tiempo, por un atribuido factor de roll-off $\alpha$. La expresión en el dominio del tiempo de la respuesta impulsiva es

$$
g_{R C}(t)=\left\{\begin{array}{cc}
1, & |t| \leq \frac{(1-\alpha) T}{2} \\
\frac{1}{2}\left[1+\cos \left(\pi P_{R C}(t)\right)\right], & \frac{(1-\alpha) T}{2}<|t| \leq \frac{(1+\alpha) T}{2} \\
0, & \text { otherwise }
\end{array}\right.
$$

El filtro de conformación de pulso de tipo RRC, se define

$$
g_{R R C}(t)=\sqrt{g_{R C}(t)}
$$

\section{Secante hiperbólica invertida (Fsech)}

El filtro de conformación de pulso de Nyquist mejorado, se propone en la [19] en el contexto de modulaciones monoportadoras, para mejorar la sensibilidad de la inestabilidad temporal. El primer filtro de conformación de pulso, considerado en la [19], es la secante hiperbólica invertida (Fsech), cuya expresión en el dominio del tiempo, se da por

$$
g_{F \text { sech }}(t)=\left\{\begin{array}{cc}
1, & |t| \leq \frac{(1-\alpha) T}{2} \\
1-\operatorname{sech}\left(\rho P_{1 F s e c h}(t)\right), & \frac{(1-\alpha) T}{2}<|t| \leq \frac{T}{2} \\
\operatorname{sech}\left(\rho P_{2 F s e c h}(t)\right), & \frac{T}{2}<|t| \leq \frac{(1+\alpha) T}{2} \\
0, & \frac{(1+\alpha) T}{2}<|t|
\end{array}\right.
$$

donde sech es la función secante, $\rho=\ln (\sqrt{3}+2) / \alpha \times T / 2, P_{1 \operatorname{sech}}(t)$ y $P_{2 \operatorname{sech}}(t)$ son los argumentos internos de la función hiperbólica escritas como

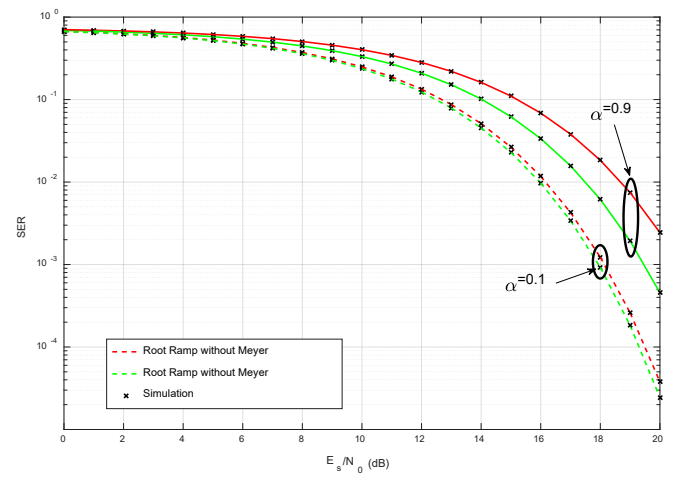

Figura 1: Rendimiento del SER del filtro de conformacion de pulso de tipo room ramp, con y sin Meyer, con valores diferentes de alfa.

\section{Flipped-inverse hyperbolic secant (Farcsech)}

Otro filtro de conformación de pulso de Nyquist mejorado, propuesta en la [19], es la secante hiperbólica flipped-inverse (Farcsech). La expresión en el dominio del tiempo del filtro a conformación de pulso Farcsech es

$$
g_{\text {Farcsech }}(t)=\left\{\begin{array}{cc}
1, & |t| \leq \frac{(1-\alpha) T}{2} \\
\operatorname{arcsech}\left(\frac{1}{\rho} P_{1 \text { Farsech }}(t)\right), & \frac{(1-\alpha) T}{2}<|t| \leq \frac{T}{2} \\
1-\operatorname{arcsech}\left(\frac{1}{\rho} P_{2 F s e c h}(t)\right), \frac{T}{2}<|t| \leq \frac{(1+\alpha) T}{2} \\
0, & \frac{(1+\alpha) T}{2}<|t|
\end{array}\right.
$$

donde Farcsech es la función secante hiperbólica inversa, $P_{1 \text { Farcsech }}(t)$ y $P_{2 \text { Farcsech }}(t)$ son los argumentos internos de las funciones secantes hiperbólicas inversas, escritas como

\section{E. Xia pulses}

Una familia de pulsos, conocida como Xia pulses, se considera en la [18]. Su principal propiedad es la de satisfacer la condición de Nyquist para una transmisión libre de interferencia entre símbolos, con y sin filtración emparejada, de este modo proporciona una mejor robustez en la instabilidad temporal. La expresión en el dominio del tiempo del filtro de conformación de Xia pulses es

$$
\begin{aligned}
& g_{\text {Xia }}(t)=\left\{\begin{array}{cc}
1, & |t| \leq \frac{(1-\alpha) T}{2} \\
\frac{1}{2}\left(1+e^{j \pi P_{1 X i a}(t)}\right), & \frac{(1-\alpha) T}{2}<|t| \leq \frac{(1+\alpha) T}{2} \\
0, & \text { otherwise }
\end{array}\right. \\
& P_{1 \text { Xia }}(t)=\left(\frac{|t|-\left(\frac{(1-\alpha) T}{2}\right)}{\alpha T}\right) .
\end{aligned}
$$

Introduciendo el concepto de función auxiliar de Meyer $v(x)$ obtenemos

$$
P_{1 \text { Xia }}(t)=v\left(\frac{|t|-\left(\frac{(1-\alpha) T}{2}\right)}{\alpha T}\right) .
$$

\section{ANÁLISIS DEL SER}

Analizamos el rendimiento del SER del sistema GFDM para transmisiones en un canal AWGN. De la [2], la expresión general para el SER de la modulación QAM es

$$
P_{A W G N}=2\left(\frac{\kappa-1}{\kappa}\right) \operatorname{erfc}(\sqrt{\gamma})-\left(\frac{\kappa-1}{\kappa}\right) \operatorname{erfc}^{2}(\sqrt{\gamma})
$$

donde $\kappa=\sqrt{2^{\mu}}$, en el cual $\mu$, es el número de bits por símbolo. Una comparación del rendimiento del SER del GFDM está hecha por filtros de conformación de pulso de Nyquist mejorado, con y sin usar las funciones auxiliares de Meyer. 
Vale la pena observar que la ecualización ZF elimina la autointerferencia en el lado del receptor, pero al mismo tiempo, introduce una intensificación del ruido. La NEF (7) depende del particular filtro de conformación de pulso. Como se muestra en la [17], la resultante equivalente del SNR es

$$
\gamma=\frac{3 R_{T}}{2\left(2^{\mu}-1\right)} \frac{E_{s}}{\xi N_{o}},
$$

donde $E_{s}$ es la energía media por símbolo, $N_{o}=2 \sigma_{w}^{2}$ es la densidad espectral de la potencia del ruido, del complejo AWGN, y $R_{T}=\frac{K M}{K M+N_{c p}+N_{c s}}$

Siendo $N_{c p}$ y $N_{s}$ la longitud del CP y el símbolo de guardia, respectivamente.

\section{RESULTADOS DE LAS SIMULACIONES}

En esta Sección realizamos simulaciones computarizadas para comparar el SER del GFDM alcanzado con diferentes respuestas impulsivas de conformación de pulso. Consideramos $K=64$ sub-portadores y $M=7$ sub-símbolos.

Ahora, analizamos el rendimiento del SER del GFDM considerando todos los pulsos en el caso del receptor ZF. La Figura 1 muestra el rendimiento del SER frente al del $E_{\mathrm{s}} / N_{0}$ en el caso de 16-QAM alcanzado por el filtro de conformación de pulso, dado en la (10), con y sin las funciones auxiliares de Meyer. Los valores del roll-off son $\alpha=0.1$ y 0.9 . De la figura observamos que la conformación del pulso con la función de auxiliar de Meyer produce el mejor rendimiento en comparación con aquella que no la tiene. Un similar comportamiento puede ser observado en la Fig. 2, por el Xia pulse en la (18), con los mismos valores del roll-off.

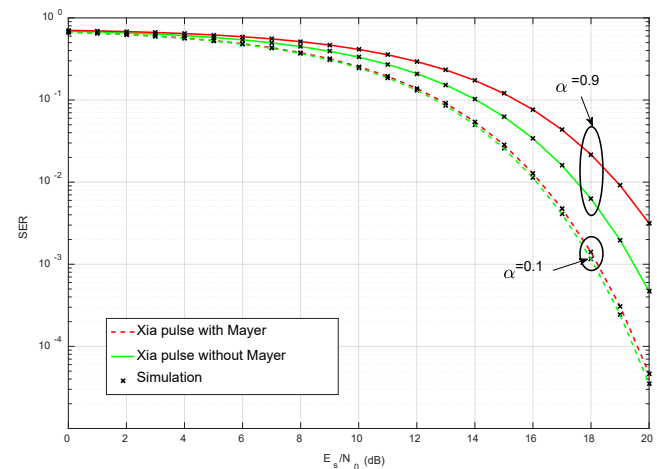

Figura 2: Rendimiento del SER del filtro de conformacion de pulso de tipo $\mathrm{Xia}$, con y sin Meyer, con valores diferentes de alfa.

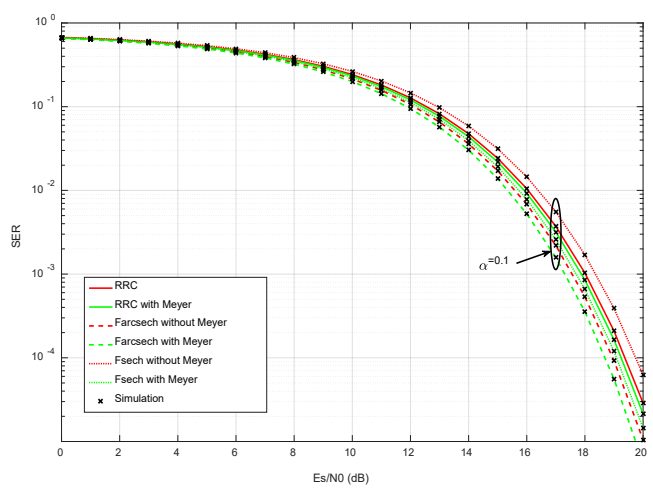

Figura 3: Rendimiento del SER con $\alpha=0.1$

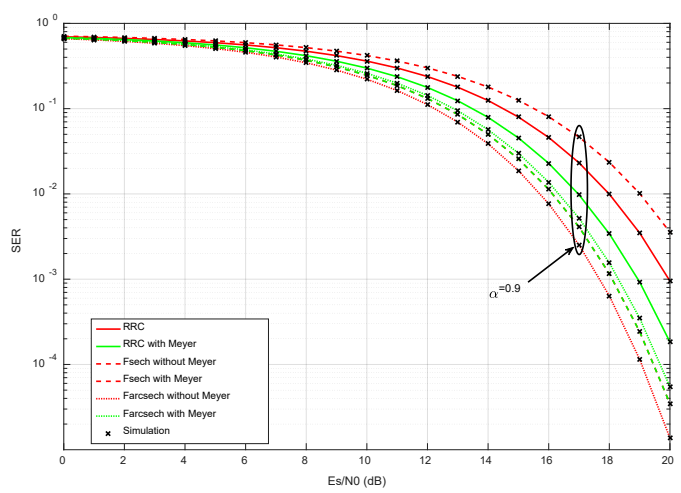

Figura 4: Rendimiento del SER con $\alpha=0.9$

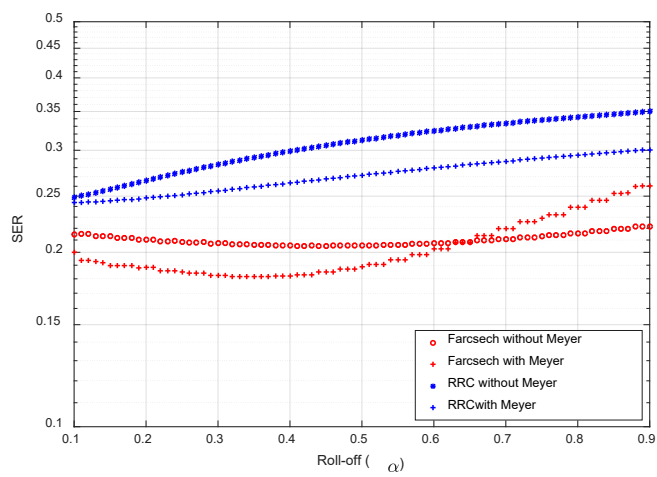

Figura 5: Comparacion del SER del RRC y del Farcsech, con y sin Meyer, con $10 \mathrm{~dB}$ de SNR.

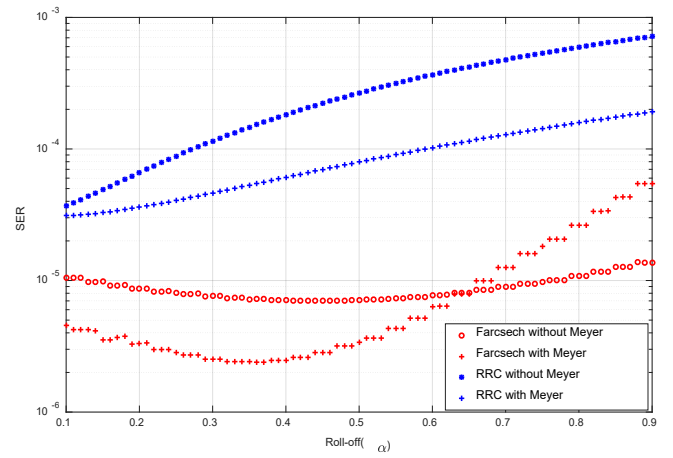

Figura 6: Comparacion del SER del RRC y del Farcsech, con y sin Meyer, con $20 \mathrm{~dB}$ de SNR

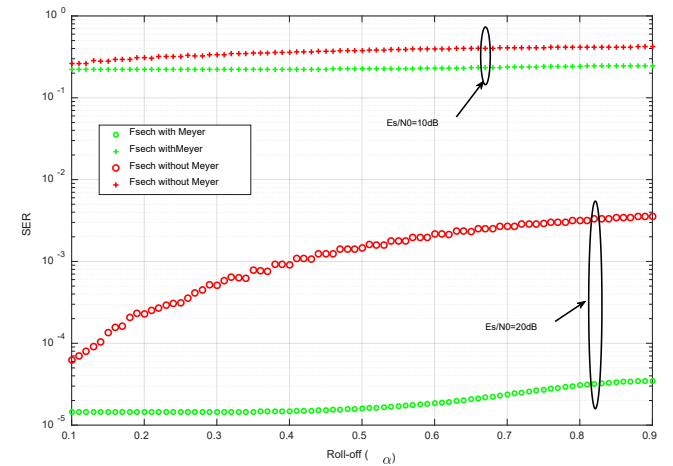

Figura 7: Comparacion del SER del Fsech, con y sin Meyer, y con valores iguales a 10 y $20 \mathrm{~dB}$ del SNR.

La Figura 3 muestra el rendimiento del SER por los filtros de conformación de pulso, dados en la [17] con y sin las funciones auxiliares de Meyer. El valor del roll-off es igual a 
0.1. En la figura observamos que el filtro de conformación de pulso de tipo Farcsech proporciona un mejor rendimiento que con el RRC, dado en la [17], y que el filtro de conformación de pulso Fsech. Además, si aplicamos el concepto de función auxiliar de Meyer en estos filtros, se observara un mejoramiento en términos de SER comparado en el caso sin función auxiliar de Meyer.

Para evaluar el impacto del valor del roll-off en el rendimiento, el mismo tipo de comparación hecho en la Fig. 3 con $\alpha=0.1$ se repite en la Fig. 4 con $\alpha=0.9$. Es claramente evidente, observando el grafico que con $\alpha=0.1$ el filtro de conformación de pulso Farcsech es todavía optimo comparado con los filtros de conformación de tipo RRC, dada en la [17], y la del tipo Fsech. Sin embargo, cuando aplicamos la función auxiliar de Meyer en el filtro de conformación de pulso, resulta un mayor SER, comparado con aquello sin Meyer.

Para investigar el efecto de la función auxiliar de Meyer en el filtro de pulsión de conformación de tipo Farcsech, en la Fig. 5 y la 6 , hemos considerado los dos valore fijos del $E_{\mathrm{s}} / N_{0}$ iguales a 10 y $20 \mathrm{~dB}$, respectivamente. Luego, hemos trazado el SER con valores diferentes de $\alpha$. Se puede observar que con valores bajos de $\alpha$, el Farcsech mejora su rendimiento con la función auxiliar de Meyer que sin ella.

Similarmente, en la Fig. 7, se muestra un SER con diferentes valores de $\alpha$, el filtro de conformación de pulso Fsech, con y sin la función auxiliar de Meyer con valores iguales a 10 y $20 \mathrm{~dB}$ del $E_{\mathrm{s}} / N_{0}$. En este caso el filtro de conformación de pulso de tipo Fsech, la función de Meyer proporciona un mejoramiento en comparación al filtro de conformación de pulso de tipo Fsech.

\section{CONCLUSION}

La multiplexación por división de frecuencia generalizada (GFDM), es un esquema de modulación multi-portadora basada en bloques, que cuenta con el uso del filtro de conformación de pulso circular en el transmisor y receptor. La GFDM es uno de los posibles esquemas de modulación bajo investigación para el nivel físico 5G. En la aplicación del GFDM varios filtros de conformación de pulso han sido propuestos, que tienen diferentes impactos en el rendimiento de la tasa de error del símbolo (SER). La principal motivación de este trabajo es la de investigar la sensibilidad del rendimiento del SER con los diferentes tipos de filtros de conformación de pulso. Nos focalizamos en los Xia pulses y también en las extensiones de esta familia de pulsos, conocidos como pulsos de "Nyquist mejorado", con funciones auxiliares de Meyer y analizando el rendimiento, en el caso de transmisión en un canal AWGN del sistema GFDM, en términos del SER en el caso del receptor zero forcing. De los resultados presentados, es claramente visible que cuando incluimos la función auxiliar de Meyer en el filtro de conformación de pulso de Nyquist mejorado, se observa un mayor mejoramiento en términos del SER.

\section{REFERENCIAS}

[1] N. Michailow, et al., "Generalized frequency division multiplexing for 5th generation cellular networks," IEEE Trans. Commun., vol. 62, no. 9 , pp. 3045-306, Sept. 2014

[2] 5GNow Deliverables online resources: http://www.5gnow.eu/wpcontent/uploads/2015/04/5GNOW D3.1 v1.1 final.pdf
[3] Y. Ding, et al., "An intelligent self-organization scheme for the internet of things," IEEE Comput. Intell. Mag., vol. 8, no. 3, pp. 41-53, 2013.

[4] G. P. Fettweis, "The Tactile Internet: Applications and Challenges," IEEE Veh. Technol. Mag., vol. 9, no. 1, pp. 64-70, Mar. 2014.

[5] N. Tadayon and S. Aissa, "Modeling and analysis of cognitive radio based IEEE 802.22 wireless regional area networks," IEEE Trans. Wireless Commun., vol. 12, no. 9, pp. 4363-4375, Sep. 2013.

[6] H. Schepker, C. Bockelmann, and A. Dekorsy, "Exploiting sparsity in channel and data estimation for sporadic multi-user communication," Intern. Symp. Wireless Commun. Systems, pp. 1-5, Aug. 2013, Germany.

[7] B. Furht and S. A. Ahson. Long Term Evolution: 3GPP LTE radio and cellular technology. Crc Press, 2016.

[8] G. Wunder, et al., "5GNOW: non-orthogonal, asynchronous waveforms for future mobile applications," IEEE Communications Magazine, vol. 52, no. 2, pp. 97-105 Feb. 2014

[9] B. Farhang-Boroujeny, "Filter bank multicarrier modulation: A waveform candidate for 5G and beyond,," Advances in Elect. Eng., 2014.

[10] V. Vakilian, et al.,"Universal-filtered multi-carrier technique for wireless systems beyond LTE," in IEEE Globecom, pp. 223-228, 2013,

[11] G. Fettweis, M. Krondorf, and S. Bittner, "GFDM - Generalized Frequency Division Multiplexing," in IEEE Vehicular tech., 2009.

[12] A. Tonello and M. Girotto, "Cyclic block FMT modulation for broadband power line communications," in IEEE Inter. Symp. on Power Line Comm. and Its Apps. (ISPLC), 2013, March 2013, pp. 247-251.

[13] P. Siohan, C. Siclet, and N. Lacaille, "Analysis and design of OFDM/OQAM systems based on filterbank theory," IEEE Trans. Signal Process., vol. 50, no. 5, pp. 1170-1183, May 2002.

[14] A. Viholainen, T. Ihalainen, T. H. Stitz, M. Renfors, and M. Bellanger, "Prototype filter design for filter bank based multicarrier transmission," IEEE Europ. Signal Process Conf, pp. 1359-1363, 2009, Glasgow, UK.

[15] M. Matthé, et al., "Influence of pulse shaping on bit error rate performance and out of band radiation of generalized frequency division multiplexing," IEEE Intern. Conf. Commun., pp. 43-48, 2014, Sydney

[16] R. Datta, N. Michailow, M. Lentmaier, and G. Fettweis, "GFDM interference cancellation for flexible cognitive radio PHY design," Vehicular Technology Conference, pp. 1-5, 2012, Quebec City, QC.

[17] A. Kumar, and M. Magarini. "Improved Nyquist pulse shaping filters for generalized frequency division multiplexing," In 8th IEEE LatinAmerican Conference (LATINCOM), pp. 1-7, 2016.

[1] C. C. Tan, and N. C. Beaulieu. "An investigation of transmission properties of Xia pulses," In Communications, IEEE International Conference ICC, vol. 2, pp. 1197-1201, 1999.

[18] A. Assalini and A. M. Tonello, "Improved Nyquist pulses," IEEE Commun. Lett., vol. 8, no. 2, pp. 87-89, Feb. 2004.

[19] I.S.Gaspar, "Waveform advancements and synchronization techniques for generalized frequency division multiplexing," 2016.

[20] 5GNow Deliverables online resources: http://www.5gnow.eu/wpcontent/uploads/2015/04/5GNOW_D3.2_final.pdf

[21] W. W. Jones and J. C. Dill, "The square root raised cosine wavelet and its relation to the Meyer functions," IEEE Trans. Signal Process., vol. 49, no. 1, pp. 248-251, Jan. 2001.

[22] I. Daubechies et al. Ten lectures on wavelets, volume 61. SIAM, 1992.

A. Kumar recibió el BSc en Ingeniería Electrónica y Comunicación en 2013

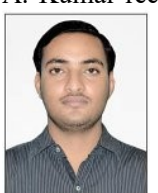
de la Univ. JAYPEE, Guna, India. En 2015, recibió el MSc en Ingeniería Electrónica y empezó su $\mathrm{PhD}$ en Telecomunicaciónes en Politecnico di Milano, Italia,. Sus intereses de investigación incluyen la comunicación inalámbrica y modulaciones de múltiples portadoras.

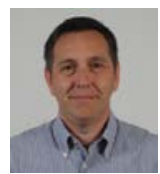

M. Magarini es Profesor Asistente en Politecnico di Milano, Italia. Es autor y coautor de más de 80 artículos de revistas y conferencias. En 2008 pasó una licencia sabática en Bell Labs, Alcatel-Lucent, Holmdel, NJ. Sus intereses de investigación incluyen la teoría de la comunicación, sincronización, estimación de canales, y codificación.

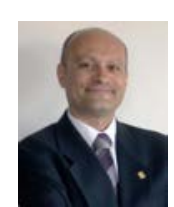

S. Bregni (SM '99) es Profesor Asociado de telecomunicaciones en Politecnico di Milano. Desde 2004 ha sido Conferencista Distinguido de la IEEE Communications Society, en la que actualmente es Vicepresidente de Miembros y Actividades Globales (2016-2017). Ha sido Co-Presidente del Programa Técnico del IEEE ICC 2016 y de varias otras conferencias. 